PAPER

\title{
Theoretical and experimental studies on the resonance frequencies of a stretched circular plate: Application to Japanese drum diaphragms
}

\author{
Hideo Suzuki ${ }^{1}$, Naoki Yamaguchi ${ }^{2}$ and Hideaki Izumi ${ }^{3}$ \\ ${ }^{1} A \& D$ Co., Ltd., \\ 1-243, Asahi, Kitamoto, 364-8535 Japan \\ ${ }^{2}$ INES Corporation, \\ 11-1, Akasaka 6-chome, Minato-ku, Tokyo, 107-8427 Japan \\ ${ }^{3}$ Faculty of Information and Computer Science, Chiba Institute of Technology, \\ 2-17-1 Tsudanuma, Narashino, 275-0016 Japan
}

(Received 2 October 2008, Accepted for publication 10 March 2009)

\begin{abstract}
Nagado-daiko, the most common type of traditional Japanese drum, is known for its unique diaphragm, which is stretched to a relatively high tension compared to other types of drums. The diaphragm is primarily made of cow skin. Once the cow skin is chemically treated, it exhibits Young's modulus of approximately $3.5 \times 10^{9} \mathrm{~Pa}$, suggesting that the diaphragm should be treated as a stretched plate, or a stiff membrane. This paper reviews the method for obtaining eigenvalues and eigen-frequencies of a membrane with stiffness. Eigenvalues of the 15 lowest modes are given as functions of tension vs. bending stiffness ratios. A double exponential function with four constants is also presented so that eigenvalues (and then resonance frequencies) of the 15 lowest modes can be simply obtained by common calculators. Measured results of a nagado-daiko with a $48 \mathrm{~cm}$ diameter show that the effect of stiffness on the spacing of resonance frequencies is negligible in the lowest several modes, contradicting theoretical prediction. A more precise experimental study is necessary to determine the effectiveness of the diaphragm stiffness on the resonance frequencies especially in the mid to high frequency region.
\end{abstract}

Keywords: Japanese drum, Circular plate, Circular membrane, Resonance frequency, Tension, Vibration mode, Stretched plate

PACS number: 43.75.Hi, 43.40.Dx [doi:10.1250/ast.30.348]

\section{INTRODUCTION}

"Nagado-daiko" (literally, "long-body drum") is the most common type of traditional Japanese drum. It has two diaphragms on both sides of its body, as seen in Fig. 1. The typical diameter of this drum ranges from 0.3 to $1 \mathrm{~m}$. The body, a hollow and circular wood trunk, is primarily made of zelkova. The diaphragm, mostly composed of cow skin, produces a large, resonant sound when struck with force. The authors examined the material properties of cow skins and observed that the skins exhibit a Young's modulus of approximately $3.5 \times 10^{9} \mathrm{~Pa}$, roughly $30 \%$ of that of zelkova. The skins' average density and thickness were measured at approximately $2,000 \mathrm{~kg} / \mathrm{m}^{3}$ and $0.002 \mathrm{~m}$, respectively [1]. Additionally, the diaphragm of the nagado-daiko is typically stretched to a higher tension than other types of drums. This paper explores how the diaphragm's large Young's modulus, as well as its tension, affects the spacing between resonance frequencies of the diaphragm and, ultimately, the tone quality of the drum.

There are many studies (for example [2-6]) in the field of vibration and sound that deal with vibrations of ideal (stiffness-free) membranes and ideal (tension-free) plates. Some of the studies consider the effect of positive and negative tensions on the resonance frequencies of a circular plate [2,5]. Reference [2] describes details of the derivation of eigenvalues (resonance frequencies) and some numerical results for various compression and tension vs. bending stiffness $(T / S)$ ratios. However, the range of the $T / S$ ratio discussed in reference [2] is too narrow to cover $T / S$ ratios of real Japanese drums (this will be made clear in Section 4).

Theoretically, the eigenvalues of the two extreme cases, i.e., the ideal plate and the ideal membrane, can be obtained by letting the $T / S$ ratio of a stretched plate equal 0 and $\infty$, respectively. In Section 2, this paper briefly reviews the theory reported in reference [2] and system- 


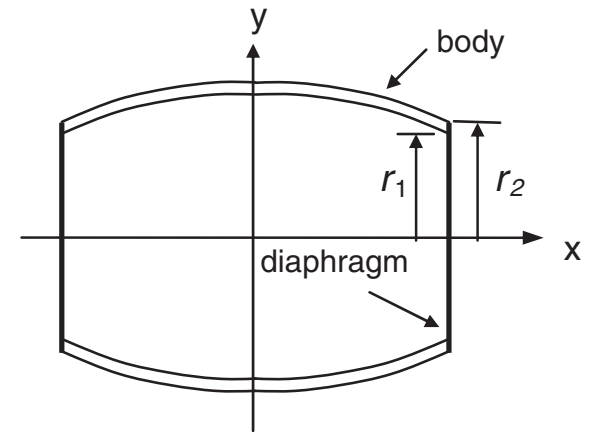

Fig. 1 Cross-section of a Japanese drum.

atically describes the determination of eigenvalues of the stretched circular plate from the ideal plate to the ideal membrane.

In Section 3, some basic properties of eigenvalues of the stretched circular plate are described, followed by a formula to calculate eigenvalues of the lowest 15 modes of a stretched circular plate for arbitrary $T / S$ ratios. Using four constants for each mode, eigenvalues (and resonance frequencies) can be calculated with just a commonly available calculator.

A more specific purpose of this study is to determine whether the diaphragm of the Japanese drum should be treated as a stiff membrane (in other words, a stretched plate) or can be treated as an ideal membrane. Very few studies have been reported on the resonance frequencies of Japanese drums [7-9]. Even though the structure of the nagado-daiko is very simple, the coupling of the two diaphragms complicates the distribution of the resonance frequencies $[10,11]$. To avoid this problem, one of the two diaphragms of a $0.48 \mathrm{~m}$ diameter nagado-daiko was removed and the resonance frequencies of the remaining diaphragm were compared to those of numerically-calculated frequencies. The details of discussion are described in Section 4 .

\section{REVIEW OF THE TRANSVERSE VIBRATION OF A STRETCHED CIRCULAR PLATE}

The theory described in this section is the same with the one given in reference [2]. Since the basic theoretical understanding of the theory is necessary for later discussions, it is briefly reviewed here. As will be shown later, the theory on a stretched circular plate can in fact cover the previously mentioned two extreme cases: an ideal plate and an ideal membrane.

\subsection{Differential Equation of the Free Transverse Vibration}

The free transverse vibration $y$ of a uniform plate with the bending stiffness $D$, the tension $T$, and the density per unit area $\rho$ is given by [2]

$$
D \nabla^{4} y-T \nabla^{2} y+\rho \frac{d^{2} y}{d t^{2}}=0
$$

where $\nabla^{2}$ is the Laplacian operator, which is defined by the equation below for the cylindrical coordinate system $(r, \theta)$.

$$
\nabla^{2}=\frac{\partial^{2}}{\partial r^{2}}+\frac{1}{r} \frac{\partial}{\partial r}+\frac{1}{r^{2}} \frac{\partial^{2}}{\partial \theta^{2}}
$$

The bending stiffness $D$ is given by

$$
D=E h^{3} /\left[12\left(1-v^{2}\right)\right]
$$

where $E$ is the Young's modulus, $h$ is the plate thickness, and $v$ is the Poisson's ratio.

\subsection{Solution of the Differential Equation}

Assuming the harmonic vibration to be $y=Y(r, \theta) e^{j \omega t}$ ( $\omega$ : angular frequency), one obtains

$$
\left(D \nabla^{4}-T \nabla^{2}-\rho \omega^{2}\right) Y=0
$$

By defining

$$
k_{1}^{2}=\frac{T+\sqrt{\left(T^{2}+4 D \rho \omega^{2}\right.}}{2 D}
$$

and

$$
k_{2}^{2}=\frac{-T+\sqrt{\left(T^{2}+4 D \rho \omega^{2}\right.}}{2 D}
$$

Eq. (4) is rewritten as

$$
\left.\left.\left[\nabla^{2}-k_{1}^{2}\right)\right]\left[\nabla^{2}+k_{2}^{2}\right)\right] Y=0
$$

The solution for Eq. (7), which is valid including $r=0$, is given by

$$
Y_{m}(r, \theta)=\left[A J_{m}\left(k_{2} r\right)+B I_{m}\left(k_{1} r\right)\right] \cos (m \theta)
$$

where $J_{m}$ and $I_{m}$ are the $m$-th order cylindrical and modified cylindrical Bessel functions, respectively.

From Eqs. (5) and (6), the relationship between $\left(k_{1} a\right)^{2}$ and $\left(k_{2} a\right)^{2}$ is obtained as

$$
\left(k_{1} a\right)^{2}=\left(k_{2} a\right)^{2}+\frac{T a^{2}}{D}
$$

where $a$ is the radius of the plate, which is introduced here for the convenience of later discussions and to make Eq. (9) dimensionless.

\subsection{Determination of Eigenvalues}

Figure 1 shows a cross-section of a Japanese drum (please be reminded that this paper considers only one diaphragm without air loading). The boundary condition of the diaphragm, however, is not easily defined due to its geometrical nonlinearity, i.e., as the diaphragm moves inward and outward, the effective radius of the diaphragm is equal to $r_{1}$ and $r_{2}$, respectively. 
To avoid the complexity of the problem, this paper analyzes eigenvalues of a stretched circular plate with the clamped (or fixed) and simply-supported conditions, hereafter abbreviated as C and SS conditions, respectively. Which of these support conditions is closer to the actual condition of the diaphragm, however, has yet to be determined.
The $\mathrm{C}$ and SS conditions at $r=a$ are defined, respectively, by

$$
Y_{m}(a, \theta)=0 \quad \text { and }\left.\quad \frac{\partial Y_{m}(r, \theta)}{\partial r}\right|_{r=a}=0
$$

and

$$
Y_{m}(a, \theta)=0 \quad \text { and } \quad M_{r}(a, \theta)=-\left.D\left[\frac{\partial^{2} Y_{m}}{\partial r^{2}}+v\left(\frac{1}{r} \frac{\partial Y_{m}}{\partial r}+\frac{1}{r^{2}} \frac{\partial^{2} Y_{m}}{\partial \theta^{2}}\right)\right]\right|_{r=a}=0
$$

where $M_{r}$ is the moment in the $r$-direction. For non-trivial solutions of $A$ and $B$ in Eq. (8), the following conditions are required for the $\mathrm{C}$ and SS conditions, respectively,

$$
\left|\begin{array}{cc}
J_{m}\left(k_{2} a\right) & I_{m}\left(k_{1} a\right) \\
\left(k_{2} a\right) J_{m}^{\prime}\left(k_{2} a\right) & \left(k_{1} a\right) I_{m}^{\prime}\left(k_{1} a\right)
\end{array}\right|=0
$$

and

$$
\left|\begin{array}{cc}
J_{m}\left(k_{2} a\right) & I_{m}\left(k_{1} a\right) \\
\left(k_{2} a\right)^{2} J_{m}^{\prime \prime}\left(k_{2} a\right)+v\left(k_{2} a\right) J_{m}^{\prime}\left(k_{2} a\right)-n^{2} v J_{m}\left(k_{2} a\right) & \left(k_{1} a\right)^{2} I_{m}^{\prime \prime}\left(k_{1} a\right)+v\left(k_{1} a\right) I_{m}^{\prime}\left(k_{1} a\right)-n^{2} v I_{m}\left(k_{1} a\right)
\end{array}\right|=0
$$

Specific values for $\left(k_{1}^{m n} a\right)^{2}$ and $\left(k_{2}^{m n} a\right)^{2}$ (eigenvalues), that satisfy Eq. (12) and Eq. (13) for the C and SS conditions can be uniquely determined once the dimensionless parameter $T a^{2} / D$ is given (remind that $\left(k_{1} a\right)^{2}$ and $\left(k_{2} a\right)^{2}$ are mutually dependent through Eq. (9)). The superscripts $m$ and $n$ give the numbers of nodal circles (excluding the boundary) and nodal lines, respectively.

Using Eqs. (5), (6) and (9), the dependence of resonance frequency on the eigenvalue $\left(k_{2}^{m n} a\right)^{2}$ is obtained as:

$$
\omega_{m n}=\left(k_{2}^{m n} a\right) \sqrt{\frac{D}{\rho a^{4}}\left[\left(k_{2}^{m n} a\right)^{2}+\frac{T a^{2}}{D}\right]}
$$

For the two extreme cases with $T a^{2} / D=0$ (ideal plate) and $T a^{2} / D=\infty$ (ideal membrane), Eq. (14) is correspondingly simplified to

$$
\omega_{m n}=\frac{\left(k_{2}^{m n} a\right)^{2}}{a^{2}} \sqrt{\frac{D}{\rho}} \quad\left(\left(k_{1}^{m n} a\right)^{2}=\left(k_{2}^{m n} a\right)^{2}\right)
$$

and

$$
\omega_{m n}=\frac{\left(k_{2}^{m n} a\right)}{a} \sqrt{\frac{T}{\rho}} \quad\left(k_{1}^{m n} a\right)^{2}=\infty
$$

Note that $\left(k_{2}^{m n} a\right)^{2}$ can be used as eigenvalues for any $T / S$ ratios including the two extreme cases. Also note that $\omega_{m n} \propto\left(k_{2}^{m n} a\right)^{2}$ and $\omega_{m n} \propto\left(k_{2}^{m n} a\right)$ in the cases of the ideal plate and the ideal membrane, respectively.

\section{EIGENVALUES OF A STRETCHED CIRCULAR PLATE}

\subsection{Dependence of Eigenvalues on the $T / S$ Ratio}

The eigenvalues are pairs of $\left(k_{1} a\right)^{2}$ and $\left(k_{2} a\right)^{2}$ with values that permit the determinant of Eq. (12) or Eq. (13)
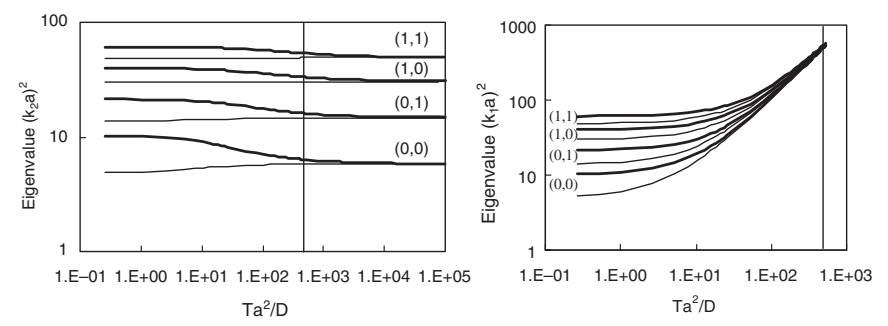

Fig. 2 Eigenvalues $\left(k_{2} a\right)^{2}$ (left) and $\left(k_{1} a\right)^{2}$ (right) of stretched circular plates with the C (thick line) and SS (thin line) conditions. The vertical line in each chart shows the position of the value of $T a^{2} / D$ of a drum used for the experiment.

to equal zero. Once $T a^{2} / D$ is given, it is possible to find these specific values of $\left(k_{1}^{m n} a\right)^{2}$ and $\left(k_{2}^{m n} a\right)^{2}$ through the conventional "convergence method." A program developed by the authors using Matlab was used to calculate these eigenvalues.

The left-side chart of Fig. 2 shows eigenvalues $\left(k_{2} a\right)^{2}$ for the $(0,0),(0,1),(1,0)$ and $(1,1)$ modes of a stretched plate as functions of $T a^{2} / D$. The thick and thin lines correspond to the $\mathrm{C}$ and SS conditions, respectively. The values of the curves as they approach their left and right ends represent those of the ideal plate and the ideal membrane, respectively. The results demonstrate that the $(0,0)$ mode has the largest percentage of change (reducing by $43 \%$ in the case of the $\mathrm{C}$ condition and increasing by $17 \%$ in the case of the SS condition) as the $T / S$ ratio increases from zero to infinity.

The right-side chart of Fig. 2 shows eigenvalues $\left(k_{1} a\right)^{2}$ in the same manner as above. As Eq. (5) shows, 
in the range where $T a^{2} / D \gg 1$, the curves are wellapproximated by $T a^{2} / D$. Eigenvalues $\left(k_{2}^{m n} a\right)^{2}$ behave better than $\left(k_{1}^{m n} a\right)^{2}$ in the sense that the former stay finite for all possible values of $T a^{2} / D$ and can be the eigenvalues for the two extreme cases (see Eqs. (15) and (16)).

The eigenvalues obtained by the program developed for this study were compared with the results for the extreme cases (ideal plate and ideal membrane) found in references [3] and [4]. Eigenvalues up to the $(3,3)$ mode are the same as those of the ideal plate given by [4] on the order of four digits when $T a^{2} / D$ is made smaller than $3 \times$ $10^{-3}$ ( $v=0.3$ in the CC condition). Eigenvalues up to the $(2,2)$ mode are the same with those of the ideal membrane given by [3] on the order of four digits if $T a^{2} / D$ is made larger than $1 \times 10^{4}$ (SS condition, $v=0.3$ ). The convergence to the ideal membrane, however, is not as precise in the case of the $\mathrm{C}$ condition. Even if the largest possible value of $3 \times 10^{5}$ is given to $T a^{2} / D$ (the program does not work for $T a^{2} / D$ values exceeding $3 \times 10^{5}$ ), there is $+0.4 \%$ error. A possible explanation for this error is that the requirement of the latter of Eq. (10) is in conflict with the ideal membrane. On the other hand, the latter of Eq. (11) is automatically satisfied when $D=0\left(T a^{2} / D=\infty\right)$. At any rate, for practical application, the error of $0.4 \%$ may not pose a problem.

Figure 3 shows $\left(k_{2}^{m n} a\right)^{2}$ of four other modes $(0,4),(1,2)$, $(1,3)$ and $(2,1)$ of stretched circular plates with the $\mathrm{C}$ (thick line) and SS (thin line) conditions. Figures 2 and 3 show that the transition region moves toward right as the eigenvalue increases, indicating that modes with larger eigenvalues behave more like a plate when $T a^{2} / D$ is kept constant.

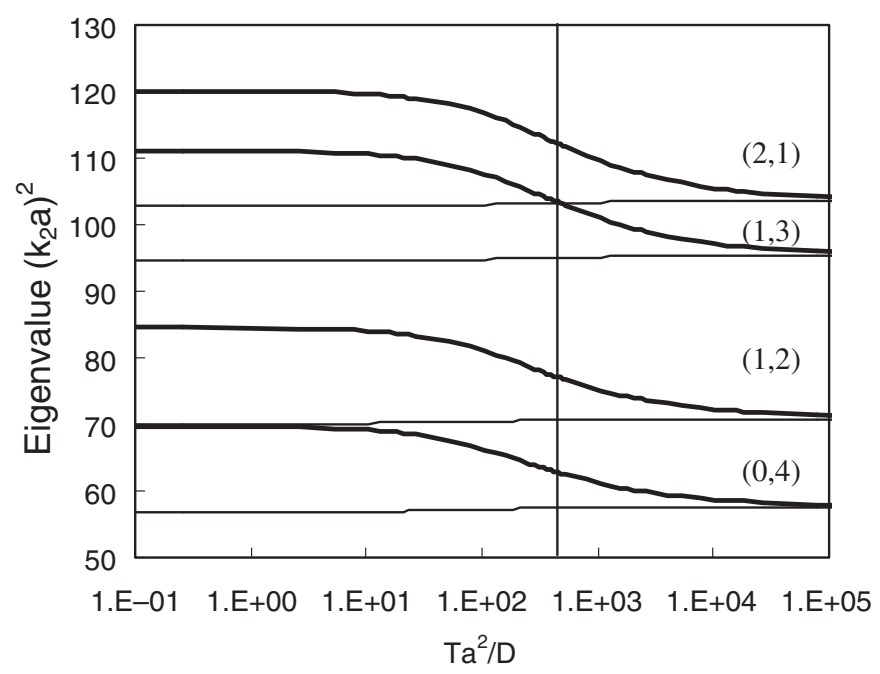

Fig. 3 Eigenvalues $\left(k_{2} a\right)^{2}$ for the modes $(0,4),(1,2)$, $(1,3)$ and $(2,1)$ of stretched circular plates with the $C$ (thick line) and SS (thin line) conditions.

\subsection{Approximation Expression of the Eigenvalues}

As mentioned before, one of the purposes of this paper is to present a method of obtaining eigenvalues for arbitrary $T / S$ ratios $\left(T a^{2} / D\right)$ of a stretched circular plate by way of simple calculation. As Figs. 2 and 3 show, the eigenvalue $\left(k_{2}^{m n} a\right)^{2}$ asymptotically approaches to one of two constants as $T a^{2} / D$ approaches to 0 or $\infty$.

The double exponential function given by Eq. (17) is used to approximate the dependence of eigenvalues $\left(k_{2}^{m n} a\right)^{2}$ on $x=\left[T a^{2} / D\right]$. This function is often used to approximate the same type of curves in various fields such as mathematics, physics, biology, and economics.

$$
f(x)=\alpha+(\beta-\alpha) \exp [-\gamma \exp \{\delta \ln (x)\}]
$$

Since

$$
f(0)=\beta
$$

and

$$
f(\infty)=\alpha
$$

$\beta$ and $\alpha$ should equal the eigenvalues of the ideal plate and ideal membrane, respectively. The remaining two parameters, $\gamma$ and $\delta$, are determined by finding a pair of $\gamma$ and $\delta$ that minimizes the mean square error between the values obtained by the theory (but numerically calculated) and approximation given by Eq. (17).

Table 1 shows the values of constants for the lowest 15 modes of stretched plates with the C and SS conditions (remember that $\alpha$ is common for both conditions). In the case of the $\mathrm{C}$ condition, the maximum errors are $-3.8 \%$ and $-3.0 \%$ for the $(0,0)$ and $(0,1)$ modes, respectively. They are $+0.9 \%$ and $+0.3 \%$, respectively, in the case of the SS condition.

The left-side and right-side charts in Fig. 4 show the "true" and approximated eigenvalues of the $(0,0)$ modes for the C and SS conditions, respectively. Large errors are observed at both ends of the transition regions. This is common for all modes discussed here even though the degrees of error are different. In the case of the SS condition, errors for all modes are less than $1 \%$, which may be small enough for practical use. As the mode numbers increase, the errors become smaller for both support conditions.

Table 1 is convenient since it gives eigenvalues of the lowest 15 modes of the ideal circular plate (both for the $\mathrm{C}$ and SS conditions) and the ideal circular membrane. The actual resonance frequencies for these cases can be calculated using Eqs. (15) or (16). If necessary, Table 1 together with Eq. (17) can be used to obtain eigenvalues for a stretched circular plate with an arbitrary $T / S$ ratio with a known maximum error. The actual resonance frequencies for this case can be calculated using Eqs. (14). 
Table 1 Constants used to obtain eigenvalues for the lowest 15 modes of stretched circular plates with the C and SS conditions for a given $T / S$ ratio by way of the approximation formula given by Eq. (17). The maximum error of approximation for each mode is also displayed.

\begin{tabular}{|c|c|c|c|c|c|c|c|c|c|}
\hline \multirow[b]{2}{*}{$(m, n)$} & \multirow[b]{2}{*}{$\alpha$} & \multicolumn{4}{|c|}{ Clamped } & \multicolumn{4}{|c|}{ Simply supported } \\
\hline & & $\beta$ & $\gamma$ & $\delta$ & $\begin{array}{l}\text { error } \\
(\%)\end{array}$ & $\beta$ & $\gamma$ & $\delta$ & $\begin{array}{c}\text { error } \\
(\%)\end{array}$ \\
\hline$(0,0)$ & 5.7832 & 10.215 & 0.1148 & 0.4868 & 3.80 & 4.9354 & 0.1735 & 0.5940 & 0.90 \\
\hline$(0,1)$ & 14.682 & 21.26 & 0.0613 & 0.5273 & 2.98 & 13.898 & 0.0843 & 0.6117 & 0.30 \\
\hline$(0,2)$ & 26.375 & 34.877 & 0.0444 & 0.5334 & 2.35 & 25.613 & 0.0612 & 0.6527 & 0.12 \\
\hline$(1,0)$ & 30.471 & 39.771 & 0.0428 & 0.5315 & 2.24 & 29.720 & 0.0418 & 0.6640 & 0.10 \\
\hline$(0,3)$ & 40.707 & 51.030 & 0.0360 & 0.5307 & 1.89 & 39.957 & 0.0325 & 0.6793 & 0.08 \\
\hline$(1,1)$ & 49.219 & 60.829 & 0.0341 & 0.5282 & 1.76 & 48.479 & 0.0272 & 0.6894 & 0.07 \\
\hline$(0,4)$ & 57.583 & 69.666 & 0.0303 & 0.5284 & 1.58 & 56.842 & 0.0243 & 0.6527 & 0.06 \\
\hline$(1,2)$ & 70.850 & 84.583 & 0.0283 & 0.5265 & 1.46 & 70.117 & 0.0207 & 0.6973 & 0.05 \\
\hline$(2,0)$ & 74.887 & 89.104 & 0.0278 & 0.5260 & 1.43 & 74.156 & 0.0199 & 0.6976 & 0.05 \\
\hline$(0,5)$ & 76.939 & 90.739 & 0.0258 & 0.5278 & 1.36 & 76.203 & 0.0197 & 0.6966 & 0.04 \\
\hline$(1,3)$ & 95.278 & 111.02 & 0.0239 & 0.5271 & 1.25 & 94.549 & 0.0172 & 0.6943 & 0.04 \\
\hline$(0,6)$ & 98.726 & 114.21 & 0.0223 & 0.5290 & 1.20 & 97.995 & 0.0170 & 0.6931 & 0.04 \\
\hline$(2,1)$ & 103.50 & 120.08 & 0.0232 & 0.5268 & 1.21 & 102.77 & 0.0164 & 0.6927 & 0.04 \\
\hline$(1,4)$ & 122.43 & 140.11 & 0.0204 & 0.5296 & 1.11 & 121.70 & 0.0152 & 0.6850 & 0.03 \\
\hline$(0,7)$ & 122.91 & 140.06 & 0.0193 & 0.5316 & 1.07 & 122.18 & 0.0150 & 0.6883 & 0.03 \\
\hline
\end{tabular}

Table 2 Measured resonance frequencies and frequency ratios of the Japanese drum diaphragm (second row) compared with the numerically obtained frequency ratios of the ideal membrane and the stretched plates with the C and SS conditions (3rd to 5 th rows). The membrane and the plates are adjusted so that they have the identical $(0,0)$ mode frequencies $(127 \mathrm{~Hz})$ with the real drum diaphragm.

\begin{tabular}{ccccccccc}
\hline Mode & $(0,0)$ & $(0,1)$ & $(0,2)$ & $(1,0)$ & $(0,3)$ & $(1,1)$ & $(0,4)$ & $(1,2)$ \\
\hline Measured & $126.9 \mathrm{~Hz}$ & $198.1 \mathrm{~Hz}$ & $271.3 \mathrm{~Hz}$ & $287.5 \mathrm{~Hz}$ & $331.3 \mathrm{~Hz}$ & $346.9 \mathrm{~Hz}$ & $378.8 \mathrm{~Hz}$ & $418.1 \mathrm{~Hz}$ \\
Ideal membrane & 1 & 1.567 & 2.138 & 2.266 & 2.611 & 2.734 & 2.985 & 3.295 \\
Stretched plate (C) & 1 & 1.593 & 2.136 & 2.295 & 2.653 & 2.917 & 3.156 & 3.500 \\
Stretched plate (SS) & 1 & 1.610 & 2.187 & 2.361 & 2.758 & 3.061 & 3.339 & 3.754 \\
\hline
\end{tabular}

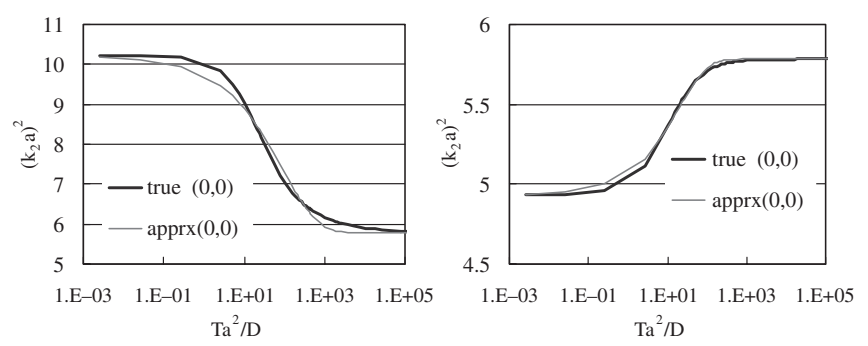

Fig. 4 Comparison of the approximated eigenvalues of $(0,0)$ modes to numerically obtained (theoretical) eigenvalues for the case of the stretched circular plates with C (left) and SS (right) conditions.

\section{COMPARISON OF CALCULATED AND MEASURED RESONANCE FREQUENCIES OF A JAPANESE DRUM DIAPHRAGM}

Another objective of this study is to determine whether the diaphragms of Japanese drums can be treated as ideal membranes, or if the bending stiffness of the diaphragms must be taken into account.
To avoid the coupling effect of the two diaphragms, a $0.48 \mathrm{~m}$ diameter Japanese drum with only one diaphragm (the other diaphragm was removed) and a $0.62 \mathrm{~m}$ long body was prepared for this study. The authors recorded the sounds that radiated from the drum when it was placed, open-side facing down, above a rigid floor and struck at a point $0.07 \mathrm{~m}$ from the edge of the diaphragm. The recording microphone was placed $0.10 \mathrm{~m}$ above the diaphragm near the striking point.

The frequency spectra of the sounds are shown in Fig. 5. The left, middle, and right charts represent the measurements for the drum when it was placed (a) directly on the floor, (b) $0.06 \mathrm{~m}$ above the floor, and (c) $0.46 \mathrm{~m}$ above the floor, respectively. It is surprising that the lowest resonance only appears when the drum is lifted from the floor (middle and the right charts). Since resonance below $100 \mathrm{~Hz}$ disappears when the drum is placed directly on the floor, it is assumed that this is not the fundamental resonance of the diaphragm. The mode numbers, then, are assigned to individual peaks as shown in the left chart.

The measured frequencies of the modes from $(0,0)$ to $(1,2)$ are shown in Table 2 (2nd row). The frequency ratios 


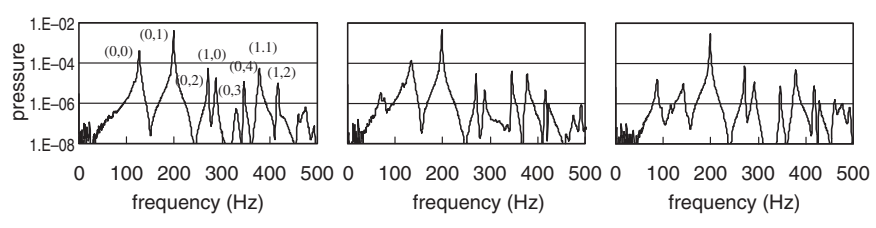

Fig. 5 Sound spectra of a drum with a single diaphragm measured when it is (a) sitting on the floor (left), (b) $6 \mathrm{~cm}$ above the floor (middle), and (c) $46 \mathrm{~cm}$ above the floor (right).

of the measured modes and those of the ideal membrane to their respective $(0,0)$ mode frequencies are also shown in Table 2 (2nd and 3rd rows). The high levels of agreement between the frequency ratios of the measured and ideal membranes in the lowest five modes (within 1.6\%) indicate that the effect of the diaphragm stiffness on the spacing of the resonance frequencies may not be significant for these lowest 5 modes.

In order to compare the resonance frequencies of the real diaphragm with the theoretical values of a stretched circular plate, the parameter $T a^{2} / D$ of the diaphragm must be known. Since the direct measurement is difficult, it must be estimated using at least one resonance frequency with known mode numbers $(m, n)$ and the diaphragm parameters such as $E=3.5 \times 10^{9} \mathrm{~Pa}, \rho=2,000 \mathrm{~kg} / \mathrm{m}^{3}, h=0.002 \mathrm{~m}$, $v=0.3$ and $r=0.23 \mathrm{~m}$ (equal to $r_{1}$ in Fig. 1). In order for the $(0,0)$ mode resonance frequency to be equal to $127 \mathrm{~Hz}$, the tension $T\left(T a^{2} / D\right)$ for the plate with the C and SS conditions must be 20,800 (429), and 23,100 (477), respectively. The positions of $T a^{2} / D=450$ $(\approx(429+477) / 2))$ on the frequency axes are marked by the vertical lines in Figs. 2 and 3. As mentioned before, the transition regions seen in Figs. 2 and 3 move toward right as the eigenvalues of modes increase. The fact that this value occurs in the transition region is an indication of the possibility that the diaphragm stiffness may influence the resonance frequency spacing in the mid to high frequency region.

The numerically obtained frequency ratios of the stretched plates with $T a^{2} / D=429$ for the $\mathrm{C}$ and with $T a^{2} / D=477$ for the SS conditions are shown in Table 2 for the lowest 8 modes and in Table 3 the for higher modes. Figure 6 shows the frequency ratios of the drum diaphragm and the stretched plates (1st, 3rd, and 4th rows of Table 3 ) to the corresponding frequencies of the ideal membrane (2nd row) as functions of the normalized ideal membrane frequency (2nd row). As the resonance frequency increases, the frequency spacing between adjacent resonances of the stretched plate becomes wider than that of the ideal membrane. Note that the stretched circular plates with the $\mathrm{C}$ and SS conditions behave similarly in terms of the frequency spacing even though they have different eigenvalues $\left(k_{2}^{m n} a\right)^{2}$ as seen in Figs. 2 and 3 around $T a^{2} / D=$
Table 3 Numerically obtained frequency ratios of the ideal membrane and the stretched plates for modes above $(2,0)$ with the $(0,0)$ mode frequency at $127 \mathrm{~Hz}$.

\begin{tabular}{cccccccc}
\hline Mode & $(2,0)$ & $(0,5)$ & $(1,3)$ & $(0,6)$ & $(2,1)$ & $(1,4)$ & $(0,7)$ \\
\hline Ideal membrane & 3.599 & 3.647 & 4.059 & 4.132 & 4.230 & 4.601 & 4.610 \\
Stretched plate (C) & 3.875 & 3.935 & 4.458 & 4.551 & 4.682 & 5.180 & 5.190 \\
Stretched plate (SS) & 3.850 & 3.909 & 4.422 & 4.515 & 4.640 & 5.130 & 5.143 \\
\hline
\end{tabular}

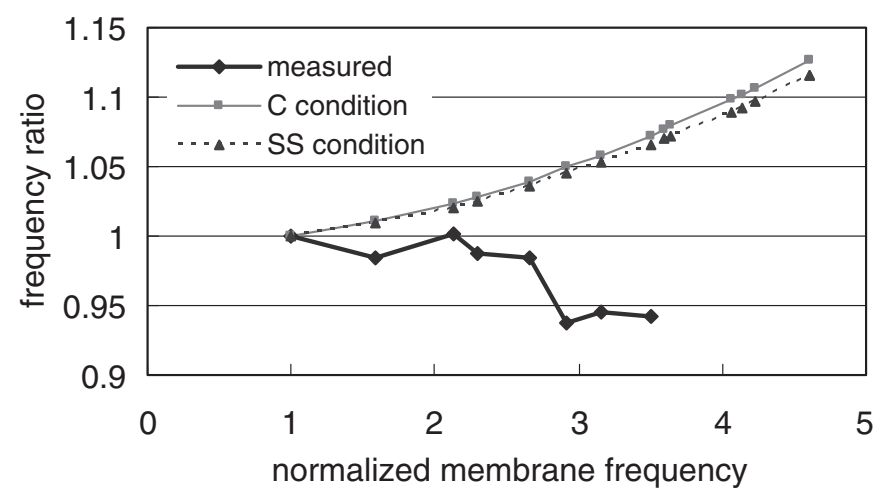

Fig. 6 Frequency ratios of the drum diaphragm (measured) and the stretched plates with the C and SS conditions (theoretical) to the corresponding frequencies of the ideal membrane (theoretical) as functions of the normalized ideal membrane frequency.

450. On the other hand, even though the corresponding eigenvalues $\left(k_{2}^{m n} a\right)^{2}$ of the stretched plate with the SS condition and the ideal membrane are close with each other, the resonance frequencies calculated using Eqs. (14) and (16) are different. For example, $\left(k_{2}^{1,2} a\right)^{2}$ of the stretched plate with $T a^{2} / D=470$ is 70.69 and that of the ideal membrane is 70.85 . The resonance frequencies of their $(1,2)$ modes, with the same $(0,0)$ mode frequency at $127 \mathrm{~Hz}$, are 473 and $442 \mathrm{~Hz}$, respectively.

What is expected for the real diaphragm from the above theoretical discussion is to show the same monotonically increasing trend the stretched plates have. On the contrary, however, the measured resonance frequencies slightly decrease as the order of modes increases at least in the lowest 8 modes. Since the effect of the stiffness is more prominent for higher modes, resonance frequencies of higher modes should be investigated. The experimental results in mid to high frequency regions, however, are harder to discuss in detail because it becomes increasingly difficult to assign mode numbers to individual resonances.

In the case of a one dimensional piano string, the degree of inharmonicity increases as the order of harmonics increases [12,13]. As seen in Fig. 6, the theoretical study of the two dimensional stretched circular plate also shows the same property (increase of resonance frequencies proportional to the square of the mode frequency). Whether or not real diaphragms own this property is 
dependent on the frequency dependence of the Young's modulus of the chemically treated cow skins.

\section{CONCLUSIONS}

This paper examined the resonance frequencies of a stretched circular plate (i.e., a stiff membrane). First, the equation of motion for a stretched circular plate and a process of obtaining eigenvalues were reviewed. The main difference between the stretched circular plate and the ideal plate is that the former has two interdependent eigenvalues for each mode. A formula with four constants was provided to obtain eigenvalues for the lowest 15 modes with arbitrary tension/stiffness ratios for each of the C and SS conditions. The formula is simple enough that even a common calculator can be used to determine the eigenvalues. Eigenvalues obtained by the formula have maximum errors of $3.9 \%$ and $0.9 \%$ in the lowest mode for the $\mathrm{C}$ and SS conditions, respectively. As the mode frequency increases, the maximum error of the approximation decreases.

In order to determine whether there is any effect of the bending stiffness on the resonance frequencies of an actual drum, resonance frequencies of a $0.48 \mathrm{~m}$ diameter drum with a single diaphragm (the second was removed to eliminate the coupling effect) were measured. As the frequency spacing in the lowest five modes was very close to that of the ideal membrane, it is suggested that the frequency spacing can be predicted by those of the ideal membrane for the several lowest modes.

One thing missing in the present theory is the effect of the radiation impedance on the resonance frequencies. Also, the non-uniformity of the diaphragm material is not taken into account. In order to better understand the effect of diaphragm stiffness on resonance frequencies, and ultimately on the tone quality, additional theoretical studies including the internal and external air loading and experimental studies using various sizes of Japanese drums are necessary.

\section{ACKNOWLEDGEMENTS}

The authors would like to thank Miyamoto Unosuke Shouten Co., Ltd., for providing the Japanese drums used in this study and for offering invaluable assistance. The authors would also like to express their gratitude to Dr. Y. F. Hwang for his cooperation on the drum study, and to Mr. Hirotaka Ozawa for the precision evaluation of the eigenvalues given by the formula. This study was carried out under a project of Acoustical Information Frontier Center, Chiba Institute of Technology.

\section{REFERENCES}

[1] N. Yamaguchi, "Study on Japanese drums -Resonance frequencies of circular membranes with bending stiffness-," Master's Thesis, Chiba Institute of Technology (2007).

[2] T. Wah, "Vibration of circular plates," J. Acoust. Soc. Am., 34, 275-281 (1962).

[3] P. M. Morse and K. U. Ingard, Theoretical Acoustics (McGraw-Hill Book Company, New York, 1968).

[4] A. W. Leissa and Y. Narita, "Natural frequencies of simply supported circular plates," J. Sound Vib., 70, 221-229 (1980).

[5] T. D. Rossing and N. H. Fletcher, "Nonlinear vibrations in plates and gongs," J. Acoust. Soc. Am., 73, 345-351 (1983).

[6] N. H. Fletcher and T. D. Rossing, The Physics of Musical Instruments (Springer-Verlag, New York, 1991).

[7] J. Obata and T. Tesima, "Experimental studies on the sound and vibrations of drum," J. Acoust. Soc. Am., 6, 267-274 (1935).

[8] T. D. Rossing, Science of Percussion Instruments (World Scientific, 2000).

[9] S. Yoshikawa et al., Acoustical Measurement of Musical Instruments, Performance, and Emotion (Corona Pub. Co., Ltd., Tokyo, 2007).

[10] H. Suzuki and Y. F. Hwang, "Coupling between two membranes of a Japanese drum," Acoust. Sci. \& Tech., 29, 215-220 (2008).

[11] Y. Hwang and H. Suzuki, "Numerical simulations of fluidstructure interactions in a Japanese drum," ICA 2007, SAV-02002-IP (2007).

[12] R. W. Young, "Inharmonicity of plain wire piano strings," $J$. Acoust. Soc. Am., 24, 267-273 (1952).

[13] H. Suzuki, "Control of inharmonicity by the addition of a point mass," J. Acoust. Soc. Jpn. (E), 10, 253-259 (1989). 